\title{
Diskussion
}

\author{
Christine Ivanov
}

\section{Transnationale Mutterschaft}

\section{Ein Literaturbericht}

Frauen machen weltweit ca. die Hälfte der Migrant/inn/en aus. In einigen Ländern, z.B. den Philippinen stellen Frauen sogar die Mehrheit der Migrierenden (vgl. Parrenas 2004). Ein großer Teil von ihnen ist ,nicht unterwegs [...], um ihre Familien wieder $\mathrm{zu}$ vereinigen, sondern um fern von ihnen eine Arbeitsstelle zu suchen" (Isaksen u.a. 2009, 56). Migrantinnen übernehmen in den Ländern der Ersten Welt häufig Tätigkeiten im Bereich der Kinderbetreuung, der Pflege von älteren Menschen oder Haushaltstätigkeiten. Sie sind überwiegend in Privathaushalten beschäftigt und dies aufgrund restriktiver Migrationspolitiken der Zielländer häufig ohne Papiere. Arbeitsschutzregelungen, feste Arbeitszeiten, angemessene Entlohnung etc. sind von ihnen nicht einforderbar, sie befinden sich in prekären Lebens- und Beschäfttigungsverhältnissen. Auf diese Aspekte der Arbeitsbedingungen in Privathaushalten, der Verwischung von Grenzen zwischen Privatheit und Öffentlichkeit oder der Verhältnisse zwischen Migrantin und Arbeitgeberin sind viele Untersuchungen zu Arbeitsmigrantinnen fokussiert. Seltener werden die Migrantinnen im Kontext ihrer Familien, die Beziehungen $\mathrm{zu}$ ihren Kindern und anderen Familienmitgliedern oder Freunden und ihre konkreten transnationalen Praktiken untersucht. Die Migration von Müttern ist in vielfältiger Weise mit ihren Familien verknüpft. Sie migrieren, um ihre Fa- milien im Herkunftsland zu unterhalten, ihren Kindern eine gute Schulausbildung zu ermöglichen oder sonstige Ziele, wie die Eröffnung eines Geschäftes, zu erreichen. Sie bleiben mit ihren Familien auf vielfaltige Weise, über gemeinsame Ziele und Projekte, regelmäßige Geldsendungen, Gespräche, Geschenke verknüpft, sie erhalten sich "gemeinsame Vorstellungswelten und Narrative der Zusammengehörigkeit" (Phoenix 2009, 86) und gehen auch weiterhin den ihnen zugeschriebenen Aufgaben als Mutter nach. Im anglo-amerikanischen Raum hat sich zur Beschreibung dieser Mutterschaftsarrangements der Begriff der ,transnational motherhood" (Hondagneu-Sotelo/Avila 1997) oder "mothering from a distance" (Parrenas 2001b, 261) durchgesetzt. Untersuchungen zu diesem Thema sind in den letzten Jahren v. a. im Kontext (kritischer) Migrationsforschung und Frauen- und Geschlechterforschung entstanden. Aber auch die Familienforschung hat das Thema furr sich entdeckt (vgl. Beck-Gernsheim 2009). Einige Forschungen nehmen ein konkretes Herkunftsland in den Blick (z.B. Horton 2008; Escriva/Skinner 2008; Schmalzbauer 2004). Und in anderen Untersuchungen zu Migration von Frauen ist transnationale Mutterschaft ein Aspekt, der neben anderen beleuchtet wird (z.B. Raijman u. a. 2003). Die Fragestellungen, Perspektiven und Vorgehensweisen der Untersuchungen variieren stark und rei- 
chen von einem feministischen Blick bis zu phänomenologischen Studien, die die intersubjektiven Erfahrungen der Mütter und Kinder betrachten. Dieser Beitrag versucht einen Überblick über den Wissens- und Forschungsstand zum Thema „transnationaler Mutterschaft“ zu bieten. Es werden solche Untersuchungen referiert und reflektiert, die sich explizit mit Mutterschaft im Kontext von Migration beschäftigten. ${ }^{1}$ Dies fuihrt zu einer gewissen Verengung, da Forschungen zu transnationalen Familienarrangements (vgl. Bryceson/Vuorela 2002; Global Networks 2005) oder zu migrierenden Vätern und Vaterschaft nur am Rande erwähnt werden (vgl. Pribilsky 2004).

\section{„Who is Taking Care of the Nanny's Children?" 2 Global Care Chains}

Transnationale Mutterschaft steht in direktem Zusammenhang mit einem Phänomen, das Arlie Hochschild (2000) als ,global care chain" bezeichnet und folgendermaBen umschrieben hat: Eine ältere Tochter aus einer armen Familie in einem Land der Dritten Welt sorgt für ihre Geschwister (das erste Glied in der Kette), während ihre Mutter als Kinderfrau arbeitet und für die Kinder einer Frau sorgt, die in ein Land der Ersten Welt migriert ist (das zweite Glied), und ihrerseits fuir das Kind einer Familie in einem reichen Land sorgt (das letzte Glied) (vgl. Hochschild 2000, 357).

Global Care Chains (Sorge- oder Betreuungsketten) können in armen Ländern beginnen und in reichen enden, sie können aber auch ländliche Regionen und

1 Wobei die Ausführungen z.T. sicher unzureichend bleiben und Lücken aufweisen, u.a. da nur deutsch- und englischsprachige Veröffentlichungen verwendet werden. In spanischsprachigen Ländern gibt es eine Reihe von Untersuchungen zur Thematik.

2 Hondagneu-Sotelo/Avila 1997, 559 städtische in dem gleichen Land verbinden oder in einem armen Land beginnen, über ein weniger armes Land erweitert werden, letztlich enden sie aber meistens in einem reichen Land (vgl. Hochschild 2000, 358). Global Care Chains überspannen nationalstaatliche Grenzen und verbinden $\mathrm{Na}$ tionalstaaten miteinander. Sie weisen auf globale Ungleichheiten auf verschiedenen Ebenen hin: zwischen armen und reichen Ländern, zwischen Frauen und Männern, in der geschlechterspezifischen Arbeitsteilung auf dem Arbeitsmarkt und in den Familien und nicht zuletzt auf intra-kategoriale Ungleichheiten zwischen Frauen. $\mathrm{Da}$ die transnationalen Migrationsbewegungen der in Betreuung und Fürsorge erwerbstätigen Frauen einseitig von den weniger entwickelten in die entwickelten Länder verlaufen, wird für die Herkunftsländer in Analogie zum Brain Drain auch von einem Care Drain gesprochen (Hochschild 2001; Hochschild/Ehrenreich 2004).

Rhacel Parrenas hat in ihren Studien zu transnationaler Mutterschaft philippinischer Hausarbeiterinnen in den USA und Italien die Auswirkungen und Effekte der transnationalen Mutterschafts- und Familienarrangements auf die migrierenden Mütter selbst, ihre Kinder, die Väter, die erweiterte Familie (Betreuungspersonen der Kinder aus dem verwandtschaftlichen Umfeld) und gesellschaftliche Vorstellungen von Familien, Mutterschaft und Geschlecht untersucht (Parrenas 2000; 2001a; 2001b; 2004; 2005a; 2005b; 2008). Sie geht von einer Globalisierung und Transnationalisierung von Reproduktionsarbeit aus, d.h. dass die historisch den Frauen zugewiesene Reproduktionsarbeit als Ware zunehmend zu einem Bestandteil des globalen Marktes geworden ist, dem sich die Entstehung von Arbeitsplätzen und wirtschaftlichem Wachstum in unterschiedlichen Regionen verdankt. Reproduktionsarbeit wird in einem mehrstufigen 
Prozess von einem Land in ein anderes transferiert: Privilegierte weiße Frauen der Mittelschicht kaufen die Arbeitskraft von weniger privilegierten Frauen und befreien sich so zumindest teilweise von ihren Verpflichtungen im Reproduktionsbereich. Die migrantischen Hausarbeiterinnen wiederum kaufen von noch schlechter gestellten Frauen, die nicht genügend Ressourcen haben, um zu migrieren, ähnliche Dienstleistungen für ihre zurückbleibenden Familien ein. In diesem „,international transfer of care taking“ (Parrenas 2000, 561) sind Frauen zwar über ihre Geschlechtszugehörigkeit und die ihnen zugeschriebenen Aufgaben miteinander verbunden, unterscheiden sich aber zugleich durch Klassenzugehörigkeit, ethnische Zugehörigkeit, Staatsbürgerschaftsstatus und die damit verbundenen Ungleichheiten voneinander (vgl. Parrenas $2000,562 \mathrm{ff}$.).

Wer die Sorge für die zurückbleibenden Kinder der migrierenden Mutter übernimmt, ist abhängig von verschiedenen Faktoren. Parrenas konnte für den philippinischen Kontext zeigen, dass Migrantinnen aus der Mittelschicht, die über qualifizierte Bildungs- und Berufsabschlüsse verfügen, vorwiegend bezahlte Hausarbeiterinnen einstellen, Frauen aus der "Arbeiterklasse“ ihre familiären Aufgaben an andere weibliche Verwandte und die älteren Töchter übertragen. Die zurückbleibenden Väter übernehmen kaum Aufgaben in der Kindererziehung- und Betreuung oder im Haushalt (vgl. Parrenas 2005b). Die Übertragung der Reproduktionstätigkeiten auf andere Frauen, weibliche Verwandte oder bezahlte Arbeiterinnen gestaltet sich oft problematisch: Weibliche Verwandte erleben die Übernahme der Aufgaben der abwesenden Mutter als eine zusätzliche Belastung zu ihren eigenen Aufgaben, die nicht mit den gleichen „Privilegien“" verbunden ist, wie sie die migrierende Mutter erhält (vgl.
Parrenas 2000, 573). Hondagneu-Sotelo und Avila (1997) beschreiben in ihrer Untersuchung zu transnationaler Mutterschaft von lateinamerikanischen Frauen in den USA, dass diese ihre Kinder bevorzugt der Obhut ihrer eigenen Mutter überlassen und so die Vorstellung reproduzieren, die biologische Mutter sorge am besten fuir ein Kind. (ebd., 559). Die Autorinnen verweisen darauf, dass es in vielen lateinamerikanischen Ländern üblich ist, sich Betreuung und Erziehung der Kinder mit anderen Frauen aus der Verwandtschaft $\mathrm{zu}$ teilen, sodass die migrierenden Frauen selten auf bezahlte Hausarbeiterinnen zurückgreifen. Sie beschreiben auch die vielfältigen Probleme und Anstrengungen, die die Mütter in diesen Arrangements erleben, gehen aber nicht auf die Perspektive der Betreuungspersonen im Herkunftsland ein. Die physisch abwesenden Mütter sorgen sich darum, ob ihre Kinder genug Zuwendung erhalten, in die Schule gehen, das Geld erhalten, welches sie senden etc. Sie engagieren sich emotional und finanziell, um ein möglichst gutes Verhältnis zu den Betreuungspersonen ihrer Kinder zu erhalten. Von ihren Arbeitgeber/innen (in den USA) erwarten sie eine reziproke Anerkennung ihrer eigenen Tätigkeit. Bleibt diese aus, führt dies zu Irritationen (vgl. Hondagneu-Sotelo/Avila 1997, 559 ff.).

Juliane Karakayali (2010) hat im Rahmen ihrer biografischen Studie zur Lebens- und Arbeitssituation von osteuropäischen Migrantinnen, die in Deutschland in Privathaushalten von pflegebedürftigen Menschen arbeiten, die transnationalen Lebensweisen der Frauen im Kontext von Mutterschaft diskutiert. Anhand eines Fallbeispiels fragt sie nach den Rollen der einzelnen, im transnationalen Familienarrangement involvierten Personen. Die Betreuung der zurückbleibenden Kinder übernehmen die Großeltern, die im Alltag die Rolle der Erziehungsberechtigten einnehmen. Die Rolle der Mutter und 
ihre Aufgaben sind in dieser Konstellation unklar und müssen immer wieder neu definiert werden. Karakayali weist darüber hinaus auf eine weitere Fragilität des Betreuungs- und Arbeitsarrangements hin: Die Frauen pflegen ältere Menschen und sind darauf angewiesen, dass ihre eigenen Eltern gesund und belastbar genug sind, um die Betreuung der Enkel zu übernehmen (vgl. Karakayali 2010, 265 ff.).

Die Einbindung in eine globale Betreuungskette kann zu Irritationen führen, die Parrenas (2000) mit „displaced mothering“ (576) umschreibt und über die auch von anderen Autorinnen berichtet wird z.B. Hondagneu-Sotelo/Avila 1997; Shinozaki 2003; Karakayali 2010). Die migrierenden Frauen sind von ihren eigenen Familien, ihren Kindern getrennt und übernehmen gleichzeitig die Sorge für die Kinder oder die Eltern Anderer. Sorge, Betreuung und Zuwendung können in diesem Kontext zu einer schmerzhaften Erfahrung werden. Auf der anderen Seite kann die Übertragung der Liebe und Sorge für die eigenen Kinder auf die der Arbeitgeber/innen aber auch zu einer Strategie werden, mittels derer die Frauen sich ihrer Liebe für die eigenen Kinder vergewissern und sich von Schuldgefuihlen entlasten können (vgl. Parrenas 2000, 576). Shinozaki (2003) beschreibt dies als eine Art Ersatzelternschaft, bei der über die Betreuungsaufgaben gegenüber Kindern in den Arbeitgeber/innenhaushalten die Liebe $z u$ den eigenen Kindern erfahren werden kann.

\section{Vertrautheit und Intimität in trans- nationalen Mutterschaftsarrangements}

Hondagneu-Sotelo und Avila (1997) verstehen unter transnationaler Mutterschaft "[...] the circuits of affection, caring, and financial support that transcend national borders [...]" (550). Bei der Betrachtung der Strategien und Praktiken, die trans- nationale Mütter anwenden, um Vertrautheit mit ihren Kindern zu erhalten und/ oder aufzubauen und herzustellen, kommen die meisten Untersuchungen zu ähnlichen Ergebnissen. Die Frauen versuchen regelmäßigen Kontakt zu ihren Kindern $\mathrm{zu}$ halten und kommunizieren per Brief und Telefon auf vielfältige Weise mit ihnen. Wenn es finanziell und aufgrund des Aufenthaltsstatus' möglich ist, besuchen die Mütter ihre Kinder und umgekehrt, Fotos und Geschenke werden ausgetauscht etc. (vgl. z.B. Hondagneu-Sotelo/Avila 1997; Lutz 2007; Parrenas 2005b). Die Frauen versuchen so intensiv wie möglich im täglichen Leben ihrer Kinder präsent $\mathrm{zu}$ sein, sich an wichtigen Entscheidungen zu beteiligen und die ihnen zugeschriebene Fürsorge- und Erziehungsverantwortung wahrzunehmen. Die physische Abwesenheit darf demnach nicht auch als emotionale Abwesenheit betrachtet werden, was in dem Zitat "I'm here, but I'm there“ (Hondagneu-Sotelo/Avila 1997, 558) einer transnationalen Mutter deutlich wird.

Die Entwicklung neuer, kostengünstiger Kommunikationstechnologien macht regelmäßige Kontakte auch über große Entfernungen hinweg einfacher. Die Internettelefonie mit einer Kamera, Mobiltelefone und SMS erlauben Müttern wie Kindern eine relativ unkomplizierte Kontaktaufnahme, die auch mehrmals täglich oder zu festen Zeiten stattfinden kann. Viele Mütter und ihre Kinder entwickeln in dieser Kommunikation gemeinsame Routinen und Rituale, die das Gefühl der Zugehörigkeit und Zusammengehörigkeit stärken (vgl. Parrenas 2005b).

Ob transnationale Kommunikation, verstanden als Fluss von Ideen, Informationen, Gütern, Geld und Gefühlen (vgl. Parrenas 2005b), stattfinden kann, in welcher Form und in welchem Ausmaß, wird von verschiedenen sozialen und geografischen Einflussfaktoren bestimmt. Kommunikation braucht Ressourcen (Kapital, Zeit, 
Infrastruktur), die jedoch ungleich verteilt sind. Für Familien, die in ländlichen Gebieten ohne eine entsprechende technologische Infrastruktur leben, ist es schwieriger, Kontakt mit der Mutter im Ausland zu halten, als für Kinder, die in einem städtischen Umfeld leben. Auch die Arbeitsbedingungen der Mutter, die je nach ihrem Aufenthaltsstatus (legal oder ohne Papiere), den Arbeitszeiten, den freien Tagen, der Lohnhöhe etc. unterscheiden, führen zu ungleichen Möglichkeiten der Kommunikation und unter Umständen zu einer unterschiedlichen Qualität der Vertrautheit in der transnationalen Mutter-Kind-Beziehung. Helma Lutz (2007), die in ihrem Buch ,Vom Weltmarkt in den Privathaushalt" auf die konkreten Alltagspraktiken transnationaler Mutterschaft eingeht und anhand zweier Fallbeispiele - eine Hausarbeiterin aus Polen und eine aus Brasilien - beschreibt, weist ebenfalls auf die vielfältigen Ungleichheiten hin, die die transnationale Kommunikation beeinflussen. Während die Hausarbeiterin aus Polen in Form der Pendelmigration jeweils einige Wochen oder Monate in Deutschland arbeitet und dann einige Zeit in Polen verbringt und ihr "die Visumsfreiheit und gute, verhältnismäßig preiswerte Verkehrsanbindungen" ein relativ hohes $\mathrm{Maß}$ an Mobilität ermöglichen, haben lateinamerikanische Frauen aufgrund ihres häufig unsicheren Aufenthaltsstatus' und der hohen Reisekosten häufig über Jahre keine Möglichkeit ihre Familien zu besuchen. (Lutz 2007, 138).

Auch Remittances, d.h., Geldüberweisungen der migrierenden Frauen an ihre Familien können als Kommunikationsmittel fungieren, welches zentrale Bedeutung für die Aufrechterhaltung von Vertrautheit und Zusammengehörigkeit haben kann. Über Remittances können die Mütter unmittelbar auf materielle Bedarfe der Familie, speziell der Kinder eingehen. Gleichzeitig weisen Investitionen etwa in
Haushaltsgegenstände darauf hin, dass die Abwesenheit der Mutter zeitlich begrenzt ist und ihre Verbindung zu einem gemeinsamen "Zuhause" weiter besteht (vgl. Parrenas 2005). Die Remittances gehen also über eine rein materielle Versorgung hinaus (vgl. Yeoh 2005). Parrenas (2005b) zeigt, dass insbesondere das Management von Bankkonten es der physisch abwesenden Mutter ermöglicht in den Alltag einbezogen zu werden. Sie entscheidet darüber, wer Zugang zu dem Geld hat und wofür es verwendet wird. Als konkrete Empfänger und Manager vor Ort setzen die Mütter häufig die ältesten Töchter ein. Sie sind für die Verteilung des Geldes verantwortlich, müssen aber auch Rechenschaft über Ausgaben geben. Parrenas sieht in der Übertragung der Verantwortung für finanzielle Fragen an die Töchter einen Indikator dafür, dass zurückgebliebene Väter in Haushaltsfragen nicht involviert sind (vgl. Parrenas 2005b, 323 ff.).

Das Erleben und die Erfahrungen der zurückbleibenden Kinder sind in den letzten Jahren vermehrt zum Gegenstand von Untersuchungen geworden. Ann Phoenix (2009) fragt in ihrer Studie mit Erwachsenen, die ,transnationale Kinder gewesen sind, danach, wie diese ,transnationale Mutterschaft und ihre eigenen Mütter beurteilen" und wie das Wiedersehen mit den Müttern war (Phoenix 2009, 88). Die Mütter waren in den 1960 er Jahren aus der Karibik nach England migriert, bis sich Mutter und Kind wiedergesehen haben, vergingen z.T. Jahrzehnte. Aufgrund der weniger entwickelten Kommunikationstechnologien waren Kontakte weniger häufig und intensiv möglich, als sie es heute sein können. In diesem Kontext weist Phoenix auf die unterschiedlichen Zeithorizonte der Mütter und Kinder hin: Die Mütter migrierten, um ihren Kindern in Zukunft ein besseres Leben zu ermöglichen. Die Kinder jedoch nahmen ihr Leben in der Gegenwart wahr und konnten 
nicht nachvollziehen, was ihre Mütter zur Migration motivierte. Die Erwachsenen waren überwiegend der Ansicht, dass die lange Trennung von der Mutter ihre Beziehungen belastet haben, was insbesondere im Zusammenhang mit dem Fehlen körperlicher Zuneigung artikuliert wird. Phoenix greift an dieser Stelle die Bedeutung populärwissenschaftliche Diskurse zu Mutter-Kind-Bindung und frühkindlichen Erfahrungen auf, die noch weiter ausgeführt werden sollten (vgl. Phoenix $2009,89 \mathrm{ff}$.).

Shinozaki (2003) stellt in ihrer Forschung fest, dass die Beziehung zwischen transnationalen Müttern und ihren Kindern intensiver und vertrauensvoller war, wenn die Kinder die Motive für die Abwesenheit der Mütter verstanden. Parrenas hat in ihren Untersuchungen mit Kindern auf den Philippinen, deren Mütter migriert sind, beschrieben, dass Kinder, die regelmäßig mit ihren Müttern telefonieren konnten, seltener einen Bruch in der Beziehung spürten. Sie nahmen die gemeinsame Gesprächzeit am Telefon als Familienzeit auf Distanz wahr und beschrieben ihre Beziehung zu der physisch abwesenden Mutter als eng, z.T. enger als die zum physisch anwesenden Vater (Parrenas 2005b, 328). Auch Lutz kommt - allerdings aus der Perspektive der transnationalen Mütter - zu dem Schluss, es gebe „zahlreiche Hinweise darauf, dass die transnationale Migration der Mütter die Beziehung zwischen Angehörigen nicht schwächt, sondern im Gegenteil festigt" (Lutz 2007, 164). Die Perspektive der zurückbleibenden Kinder, transnationale Kindheit und wie diese erlebt wird, mit welchen Herausforderungen sie für das Kind ist verbunden ist, wie transnationale Kommunikation erlebt wird oder welche (Aus-) Wirkungen die Erfahrungen auf eigene Vorstellungen von Elternschaft und Familie haben, gilt es noch stärker zu betrachten.

\section{Transnationale Mutterschaft im Kontext ideologischer Vorstellungen von Mutterschaft}

Sigrid Metz-Göckel, Senganata Münst und Dobrochna Kalwa (2010) beschreiben in ihrer multi-lokal angelegten Studie zur Lebens- und Arbeitssituation von polnischen Hausarbeiterinnen in Deutschland u.a. ausführlich den Migrationsdiskurs in Polen, der sich mit der Abwesenheit von Frauen/Müttern und deren angeblich negativen Folgen für Familie und Kinder befasst, während migrierende Männer/ Väter nur am Rande thematisiert werden. Die Autorinnen sprechen von einer „Pathologisierung unvollständiger Migrationsfamilien", die sich in erster Linie gegen die Frauen/Mütter wendet. Kalwa geht genauer auf die Rolle der katholischen Kirche ein, die in Polen einen erheblichen Einfluss auf die öffentliche Meinung ausübt: In Hirtenbriefen und Flugblättern wird vor der Migration der Mütter und deren Gefahren und negativen Folgen für die zurückbleibende Familie gewarnt; den Kindern fehle der emotionale Halt und die Frauen entfremdeten sich von ihren Ehepartnern (Metz-Göckel u.a. 78 f.). Die in der Untersuchung befragten Frauen stellten die normativen Vorstellungen von Familie und den Aufgaben der Frauen selten in Frage. Kalwa schließt daraus, dass die Frauen ihre Selbstbildnisse entsprechend vorherrschender Geschlechterbilder und Normen aufrecht zu erhalten bzw. aktiv zu konstruieren versuchen. Eine Infragestellung oder gar Neudefinierung von Geschlechterrollen und -normen oder der Selbstbildnisse der Frauen kann Kalwa nicht erkennen (vgl. Metz-Göckel u.a. 2010, $101 \mathrm{f}$.).

Die Rolle, die der Institution Kirche und religiösen Vorstellungen und Netzwerken sowohl in den Herkunfts- als auch in den Zielländern bei der Konstruktion von Mutterschaftsidealen zukommt, wurde 
im Kontext transnationaler Mutterschaft bisher nur selten untersucht: HondagneuSotelo/Avila (1997) fragen nach der symbolischen Bedeutung von katholischen Heiligenfiguren und der Jungfrau Maria, für Vorstellungen von Mutterschaft. Sie gehen davon aus, dass traditionelle Konzeptionen von Familie und Mutterschaft in Lateinamerika stark von den Ideologien der katholischen Kirche geprägt sind (ebd., 551).

Parrenas (2005a, $30 \mathrm{ff}$.) reflektiert ausführlich die diskursive Herstellung und Reproduktion von traditionellen Geschlechtsrollen und Familienvorstellungen auf den Philippinen. Sie betrachtet rechtliche Regelungen die Familie betreffend, mediale Auseinandersetzungen mit dem Thema abwesender Eltern im Kontext von Migration und die Rolle weiterer gesellschaftlicher Institutionen, zum Beispiel der Schule oder verschiedener Nichtregierungsinstitutionen, die sich für $\mathrm{Mi}$ grant/inn/en einsetzen. Diese idealisieren das für die Philippinen als traditionell konstruierte Familienbild der Kernfamilie mit einem männlichen Haushaltsvorstand und Familienernährer und der Mutter als voranging zuständig für alle Reproduktionstätigkeiten. Andere Familienformen, insbesondere transnationale mit einer abwesenden Mutter werden stigmatisiert.

Auch akademische Auseinandersetzungen mit transnationaler Mutterschaft konstruieren ein z.T. sehr negatives Bild dieser Familienarrangements. Sarah Horton, die undokumentierte transnationale Mütter aus El Salvador in den USA befragt hat, kritisiert, dass Studien häufig der Lebenswirklichkeit der Frauen nicht gerecht werden: „In privileging the exploration of transnational separations through the single lense of gender, such researches reduces the embodied distress of mothers and children [...]" (Horton 2009, 21). Sie geht insbesondere der Frage nach, wie transnationale Mutterschaft intersubjektiv von den Frauen und ihren Kindern erlebt wird. Sie weist jegliche „Feier“ von entstehenden transnationalen Räumen zurück und kommt zu dem Schluss, dass transnationale Mutterschaft durch vielfältige Immobilität gekennzeichnet ist, unter der die betroffenen Mütter sehr stark leiden (vgl. Horton 2009).

Deutlich wird, dass transnationale Mütter vielfach Kritik und Schuldzuweisungen ausgesetzt sind. Sie müssen ihre Entscheidung zur Migration vor sich selbst, ihren Kindern, anderen Familienmitgliedern und der Gesellschaft rechtfertigen. Ihnen wird häufig abgesprochen eine gute Mutter zu sein. Hondagneu-Sotelo/Avila (1997) beschreiben diese Situation folgendermaßen: „When men come north and leave their families [...] they are fulfilling familial obligations defined as breadwinning for the family. When women do so, they are embarking not only on an immigration journey but on a more radical gendertransformative odyssey. [...] they must cope with stigma, guilt, and criticism from others“ (ebd., 552).

An dieser Stelle wird die Wirkungsmacht von traditionellen Vorstellungen und diskursiven Aushandlungen zu Geschlecht, Geschlechterverhältnissen und Mutterschaft sehr deutlich, aber auch das emanzipatorische Potenzial transnationaler Mutterschaft, im Sinne einer Veränderung von festgeschriebenen Rollen, Positionierungen und Beziehungen, wird erkennbar. Dieses Potenzial und die Herausforderungen sind im Kontext der in den Herkunftsländern der Frauen vorherrschenden Mutterschaftsdiskurse zu betrachten, wozu die Einnahme einer transnationalen Perspektive in der theoretische Verortung aber auch in der Forschungspraxis nötig ist. 


\section{Transnationale Mutterschaft als} Herausforderung für die Geschlechterverhältnisse?

Die beschriebenen Herausforderungen durch die Migration von Müttern, die ihre Kinder im Herkunftsland zurücklassen, können ein Re-Arrangement von und in Haushalten und Familien veranlassen und unter Umständen zu einer Umund/oder Neu-Ordnung von hegemonialen Geschlechterverhältnissen in den Familien führen. Diese Perspektive wird in verschiedenen Untersuchungen unterschiedlich diskutiert. Lutz (2007) geht in ihrer Untersuchung unter anderem der Frage nach, „ob und in welchem Ausmaß sich [...] hierarchische Beziehungen zwischen den Geschlechtern [...] verändern oder eher verfestigen [...]“ (ebd., 126). Sie kommt zu dem Schluss, dass die Migration von Müttern zum Teil mit Verschiebungen $\mathrm{zu}$ Ungunsten der Frauen verbunden ist. Die Frauen verlassen die Diskurse um traditionelle Mutterschaft nicht, sondern verschieben sie allenfalls an einigen Stellen. Sie erweitern ihre Praktiken von Mutterschaft insbesondere um die Rolle der Familienernährerin. Lutz zufolge entsteht daraus auch eine Krise des männlichen Selbstverständnisses: Die zurückbleibenden Väter können ihre traditionelle Rolle nicht mehr wahrnehmen, vollziehen aber auch keinen Rollenwechsel, bei dem sie die bisher als weiblich konnotierten Aufgaben wahrnehmen würden. Anders als Pribilsky (2004), der in seiner multi-lokalen Untersuchung mit transnationalen $\mathrm{Mi}$ granten aus Ecuador in den USA, Männer im Kontext von Familie, Partnerschaft und Vaterschaft untersucht hat, kann Lutz ihre Feststellung empirisch aber an dieser Stelle nicht belegen.

Rhacel Parrenas geht davon aus, dass transnationale Mutterschaft keine radikalen Veränderungen in den Geschlechterverhältnissen hervorruft, vielmehr ist ihr zufolge ein Zusammenspiel von Aufrechterhaltung und Anfechtung von Geschlechternormen und -rollen $\mathrm{zu}$ beobachten. Die migrierenden Mütter geben die ihnen zugeschriebenen geschlechtsspezifischen Aufgaben und Verantwortlichkeiten nicht $a b$, wenn sie ihre Kinder verlassen. Sie versuchen weiterhin die ihnen zugewiesene Erziehungsverantwortung wahrzunehmen. Paradoxerweise tragen auch die modernen Kommunikationstechnologien in gewisser Weise zur Aufrechterhaltung von traditionellen Geschlechtervorstellungen bei, da sie Erziehung und Sorge für die Kinder auch über weite Distanzen hinweg ermöglichen. Die Rolle der Mutter wird zwar um die ökonomische Verantwortung erweitert und der Mythos des männlichen Familienernährers wird in Frage gestellt. Da die zurückbleibenden Väter aber die Übernahme weiblich konnotierter Aufgaben ablehnen, und diese von den migrierenden Frauen an andere Frauen übertragen werden, wird die normative Geschlechterordnung sogar verstärkt (vgl. Parrenas 2005a).

Shinozaki (2003) setzt sich in ihrer Untersuchung mit transnationalen Müttern und Vätern aus den Philippinen, die in Deutschland als Hausarbeiter/innen tätig sind, mit der Frage auseinander, wie Migrant/inn/en ihre transnationale Elternschaft erleben und wie möglicherweise ,Identitäten als Mütter oderVäter in Folge der Veränderung der idealtypischen Geschlechterrollen durch Arbeitsmigration rekonstruiert oder verhandelt" werden (Shinozaki 2003, 68). In ihrer Studie wird deutlich, dass es Unterschiede in der Wahrnehmung der Trennung von Kindern bei Müttern undVätern gibt. Großen Einfluss haben hierbei gesellschaftlich konstruierte Erwartungen an Eltern. Vor allem die Mütter leiden unter der Trennung von ihren Kindern, wenn sie an den normativen Erwartungen an sich selbst festhalten und so das Gefühl bekommen, ihre Rolle 
als Mutter nicht zu erfüllen. Shinozaki zeigt aber auch Beispiele auf, in denen ein „kreatives Arrangement" (Shinozaki 2003, 81) praktiziert wird und sich das Selbstverständnis der Frauen nicht auf das der idealtypischen Mutter beschränkt. Sie beschreibt eine Familie, in der der zurückgebliebene Vater eine Art Vermittlerfunktion eingenommen hat und "die Kinder so erzogen hat, dass sie den Grund der Abwesenheit der Mutter verstehen konnten" und andere Gemeinsamkeiten, als die des physischen Beisammenseins zur Grundlage ihrer Beziehung machen konnten. Als eine Gemeinsamkeit wird das „Frausein“ beschrieben (vgl. Shinozaki 2008, 81).

\section{Versuch eines Ausblicks}

Transnationale Mutterschaft bedeutet mehr als nur, dass die eigenen Kinder in einem anderen Land aufwachsen. Transnationale Mutterschaft ist verbunden mit Veränderungen von traditionellen Vorstellungen von Mutterschaft und dies auf verschiedenen Ebenen: Zunächst müssen die transnationalen Mütter selbst neue Definitionen für ihre Mutterschaft finden, sie müssen ihre Integrität als Mutter immer wieder neu herstellen. Transnationale Mutterschaft und damit verbundene alternative Definitionen davon, was Mutterschaft ausmacht und bedeutet, können wiederum Einfluss auf das Verständnis von Mutterschaft in den Herkunfts- als auch den Zielkontexten der Migrantinnen haben. Eine Veränderung von traditionellen Vorstellungen von Mutterschaft oder gar eine Dynamisierung oder Transformation von Geschlechterverhältnissen in den Herkunftsländern können die beschriebenen Studien jedoch, wenn überhaupt, nur in Einzelfällen ausmachen. Transnationale Migration stellt einen Ort der ständigen Aushandlung über ambivalente gesellschaftliche und familiäre Erwartungen an die Frau und dem Streben nach Autonomie und Handlungsmacht dar und ermöglicht eine Erweiterung der Subjektposition der Migrantinnen (vgl. Yeoh 2005). Diese Perspektive wird in den beschriebenen Studien allerdings nur an wenigen Stellen aufgegriffen.

Stärker in den Blick zu nehmen sind auf der einen Seite die Perspektiven und Positionen der zurückbleibenden Familienangehörigen, der Kinder, der Väter und derjenigen, die die Aufgaben der Mütter während ihrer Abwesenheit übernehmen, und gleichzeitig, wie auch Shinozaki (2003) fordert, wäre es wichtig zu analysieren, welche Wechselwirkungen die transnationalen Familienarrangements auch auf die Arbeitgeber/innen-Haushalte in den Zielländern der Migrantinnen haben.

Wenig reflektiert wird in den beschriebenen Studien die potenzielle politische Brisanz der Ergebnisse. Untersuchungen, die zeigen, dass sowohl die Migrantin als auch die zurückbleibenden Kinder unter dem transnationalen Mutterschaftsarrangement leiden, können leicht Argumente für Forderungen nach einer Rückkehr der Frau ins Haus liefern und dementsprechend politisiert werden. Auch Migrationspolitiken der Ziel- aber z.T. auch bzgl. der Herkunftsländer, die ganz entscheidend zur Entstehung transnationaler Familien beitragen, sollten differenzierter im Kontext von Familien (-politik) betrachtet werden. Bezogen auf den deutschsprachigen Kontext fehlen ebenfalls Untersuchungen, die die Ebene der Repräsentation in den Blick nehmen oder die mediale Auseinandersetzungen mit der Thematik betrachten.

Alle beschriebenen Studien analysieren transnationale Mutterschaft im Kontext von Arbeitsmigration im Bereich domestic work und der damit in Zusammenhang stehenden Transnationalisierung sozialer Ungleichheit. Dieser Blick sollte ausgeweitet werden auf andere Bereiche, z.B. 
auf transnationale Mutterschaft im Kontext globalisierter Sexarbeit. Aber auch die internationale Mobilität etwa von Wissenschaftlerinnen kann zur Entstehung transnationaler Familienbeziehungen beitragen, wenn fehlende institutionelle und soziale Kinderbetreuungsmöglichkeiten in den (vorübergehenden) Zielländern eine $\mathrm{Mi}$ gration gemeinsam mit Kindern unmöglich machen.

Der Fokus der Betrachtung transnationaler Mutterschaft ist bisher auf Situationen beschränkt, in denen die Migrantinnen in prekären Arbeitsverhältnissen tätig sind. Aber ,(t)he ideal of biological mothers raising their own children is widely held but is also widely broken at both ends of the class spectrum. Wealthy elites have always relied on others - nannies, governesses, and boarding schools - to raise their children" (HondagneuSotelo/Avila 1997, 557). Beck-Gernsheim (2009) beschreibt die Praktik wohlhabender Eltern aus Südostasien, die ihre Kinder zum Schulbesuch in die USA oder nach Kanada schicken. Es wäre interessant sich auch dieses andere Ende des Klassenspektrums hinsichtlich der Praktiken und Wahrnehmung von Mutterschaft anzuschauen und zu analysieren, welche Transformationen der Geschlechterverhältnisse und Mutterschaftsvorstellungen hierbei auf wen zurückwirken.

\section{Literatur}

Beck-Gernsheim, Elisabeth (2009): Ferngemeinschaften. Familien in einer sich globalisierenden Welt. In: Burkart, Günter (Hrsg.): Zukunft der Familie. Prognosen und Szenarien. Opladen, S. 93-109.

Bryceson, Deborah/Vuorela, Ulla (Hrsg.) (2002): The Transnational Family. New European Frontiers and Global Networks. Oxford.

Burkart, Günter (Hrsg.) (2009): Zukunft der Familie. Prognosen und Szenarien. Opladen.
Ehrenreich, Barbara/Hochschild, Arlie Russell (Hrsg.) (2004): Global Woman. Nannies, Maids, and Sex Workers in the New Economy. New York.

Escriva,Angele/Skinner, Emmeline (2008): Domestic Work and Transnational Care Chains in Spain. In: Lutz, Helma (Hrsg.): Migration and Domestic Work. A European Perspective on a Global Theme. Aldershot, S. 113-126.

Gamburd, Michele (2004): Breadwinner No More. In: Ehrenreich, Barbara/Hochschild, Arlie Russell (Hrsg.): Global Woman. Nannies, Maids, and Sex Workers in the New Economy. New York, S. 190-206.

Hochschild, Arlie Russell (2000): The Nanny Chain. In: The American Prospect, Jg. 11, H. 1, S. 1-4.

Hondagneu-Sotelo, Pierrette/Avila, Ernestine (1997): I'm here, but I'm there. The meanings of Latina transnational motherhood. In: Gender \& Society, Jg. 11, H. 5, S. 548-571.

Horton, Sarah (2009): A Mother's Heart is Weighed Down with Stones: A Phenomenological Approach to the Experience of Transnational Motherhood. In: Culture, Medicine, and Psychiatry, Jg. 33, H. 1, S. 21-40.

Karakayali, Juliane (2010): Transnational Haushalten. Biografische Interviews mit care workers aus Osteuropa. Wiesbaden.

Lutz, Helma (2007): Vom Weltmarkt in den Privathaushalt. Die neuen Dienstmädchen im Zeitalter der Globalisierung. Opladen.

Lutz, Helma (Hrsg.) (2008): Migration and Domestic Work. A European Perspective on a Global Theme. Aldershot,

Lutz, Helma (Hrsg.) (2009): Gender Mobil? Geschlecht und Migration in transnationalen Räumen. Münster.

Metz-Göckel, Sigrid/Münst, Senganata/Kalwa, Dobrochna (2010): Migration als Ressource. Zur Pendelmigration polnischer Frauen in Privathaushalten der Bundesrepublik. Opladen.

Parreñas, Rhacel S. (2000): Migrant Filipina Domestic Workers and the International Division of Reproductive Labour. In: Gender E Society,Jg. 14, H. 4, S. 560-580.

Parreñas, Rhacel S. (2001a): Servants of Globalization. Women, Migration and Domestic Work. Stanford, California. 
Parreñas, Rhacel S. (2001b): Mothering From a Distance: Emotions, Gender, and Intergenerational Relations in Filipino Transnational Families. In: Feminist Studies, Jg. 27, H. 2, S. 361-390.

Parreñas, Rhacel S. (2003): Servants of Globalization. Women, Migration and Domestic Work. Stanford. California.

Parreñas, Rhacel S. (2004): The Care Crisis in the Philippines: Children and Transnational Families in the New Global Economy. In: Ehrenreich, Barbara/Hochschild, Arlie Russell (Hrsg.): Global Woman. Nannies, Maids, and Sex Workers in the New Economy. New York, S. 39-54.

Parreñas, Rhacel S. (2005a): Children of Global Migration. Transnational Families and Gendered Woes. Stanford, California.

Parreñas, Rhacel S. (2005b): Long distance intimacy: class, gender and intergenerational relations between mothers and children in Filipino transnational families. In: Global Networks, Jg. 5, H. 4, S. 317-336.

Parreñas, Rhacel S. (2008): Perpetually Foreign: Filipina Migrant Domestic Workers in Rome. In: Lutz, Helma (Hrsg.): Migration and Domestic work. A European Perspective on a Global Theme. Aldershot, S. 99-112.

Phoenix, Ann (2009): Idealisierung emotionaler Bindung oder materielle Versorgung? Transnationale Mutterschaft und Kettenmigration. In: Lutz, Helma (Hrsg.): Gender Mobil? Geschlecht und Migration in transnationalen Räumen. Münster, S. 86-101.
Pribilsky, Jason (2004): ,Aprendemos a convivir: conjugal relations, co-parenting, and family life among Ecuadorian transnational migrants in New York City and the Ecuadorian Andes. In: Global Networks, Jg. 3, H. 4, S. 313-334.

Raijman, Rebecca/Schammah-Gesser, Silvina/ Kemp, Adriana (2003): International Migration, Domestic Work, and Care Work. Undocumented Latina Migrants in Israel. In: Gender E Society, Jg. 17, H. 5, S. 727-749.

Schmalzbauer, Leah (2004): Searching for wages and mothering from Afar: The case of Honduran transnational families. In: Journal of Marriage and Family, Jg. 66, H. 5, S. 1317-1331.

Shinozaki, Kyoko (2003): Geschlechterverhältnisse in der transnationalen Elternschaft. Das Beispiel philippinischer HausarbeiterInnen in Deutschland. In: beiträge zur feministischen theorie und praxis, Jg. 26, H. 62, S. 67-86.

Widding Isaksen, Lise/Sambasivan Devi, Uma/ Hochschild, Arlie (2009): Die globale Fürsorgekrise. In: WestEnd, Jg. 6, H. 2, S. 56-79.

Yeoh, Brenda/Huang, Shirlena/Lam, Theodora (2005): Transnationalizing the ,Asian' family: imaginaries, intimacies, and strategic intents. In: Global Networks, Jg. 5, H. 4, S. 307-315. 